\title{
Todesfälle bei Rauscherleben durch Butangasinhalation
}

Heinrich Elsner

\author{
Death in Ecstasy due to Butane Gas Inhalation
}

\section{Zusammenfassung}

Butangas ist ein Gas, das wegen seiner fehlenden bzw. geringen Toxizität in vielen Bereichen des täglichen Lebens eingesetzt werden kann. Demgegenüber stehen Todesfälle nach Butangasinhalation zur Rauscherzeugung. Auf den ersten Blick sind diese beiden Feststellungen unvereinbar.

Der vorliegende Aufsatz stellt eine Arbeitshypothese zu einem eventuell möglichen gemeinsamen Pathomechanismus des Rauscherlebens und der Todesfälle zur Diskussion.

\section{Schluiisselwörter}

Schnüffelstoffe · Lachgas · Butangas · (zerebrale) Hypoxie · autoerotische Stimulation

\section{Abstract}

Butane is a gas which is used in many spheres of daily life because of its missing respectively minimal toxicity. In contrast exists death after butane gas inhalation for having ecstasy. At first sight these both statements are incompatible.

This essay discusses a hypothesis for a potentially common pathomechanism of ecstasy and of death. The responsible pathomechanism is the hypoxia, which created at first the ecstasy (well-known from autoerotic stimulation) and later on the damage to the essential structures of brain and heart.

Key words

Inhalant abuse - butane $\cdot$ nitrous oxide $\cdot($ cerebral) hypoxia $\cdot$ autoerotic stimulation

\section{Darstellung des Problems}

1998 starb ein jugendlicher Leistungssportler in Essen, nachdem er Butangas aus einer Kartusche für Campinggaskocher inhaliert hatte. Am Auffindungsort der Leiche zeigte sich, dass der Jugendliche mit der Nase und dem Mund über dem Loch der Kartusche lag. Der Jugendliche hatte das Gas inhaliert, um ein Rauscherlebnis zu erlangen.

Anfang 2001 starb in Gelsenkirchen ein 13-jähriger Schüler infolge einer Butangasinhalation wahrscheinlich aus einem Gasfeuerzeug. Die Nachforschungen der Polizei ergaben, dass der Schüler bereits seit dem 11. Lebensjahr Schnüffelstoffe konsumierte. Der Autopsiebericht wies auf Organveränderungen hin, die nur bei wiederholtem Schnüffeln auftreten. Die Nachforschungen der Po- lizei im Umfeld des Schülers zeigten, dass das Inhalieren von Butangas zum Zwecke der Rauscherzeugung keine Seltenheit ist, eine genaue Anzahl der Konsumenten war nicht zu ermitteln. Als Inhalationsmechanismus wurde eruiert, dass das Gasfeuerzeug bzw. die Feuerzeugnachfüllflasche geöffnet und das ausströmende Gas inhaliert wird. Alternativ wird das Flüssiggas auf ein Kleidungs- oder Stoffstück geträufelt, dies vor Mund und Nase gepresst („huffing“) und das dann von dort aus verdampfende Gas inhaliert. Dabei wird im Allgemeinen durch mechanische Hilfsmittel versucht, die Konzentration des Butangases zu maximieren und damit die der normalen Einatemluft zu minimieren. Aus den geschilderten Konsummechanismen ist zu entnehmen, dass eine zu geringe Butangaskonzentration kein Rauscherleben hervorruft. Als Inhalt des Rauscherlebens wurde von den Schülern ein intensives sexuell akzentuiertes Gefühlsereignis angegeben. 
Ende 2001 verstarb eine 14-jährige Schülerin in Karlsburg/Mecklenburg-Vorpommern; sie hatte Butangas in eine kleine Plastiktüte geleitet und daraus inhaliert. Nach der Inhalation verlor sie das Bewusstsein, 2 Tage später verstarb sie im Krankenhaus. Auch bei ihr ergab die Autopsie Hinweise auf bereits länger bestehenden Schnüffel-Konsum. Nach Informationen der Polizei dort sei das Schnüffeln ein sehr großes Problem unter den Schülern; kurz nach diesem Zwischenfall sei ein weiteres Kind mit einem Schnüffel-Zwischenfall in das Krankenhaus eingeliefert worden.

Im September 2002 konsumierten 6 Kinder im Alter von 13-16 Jahren in Tarp/Schleswig-Holstein Butangas; eines starb unmittelbar, die anderen mussten intensivmedizinisch behandelt werden.

Aus dem Internet sind weitere Todesfälle in Deutschland durch Butangasinhalation zu entnehmen:

1996 starb ein 15-jähriger Junge an den Folgen der Butangasinhalation (wahrscheinlich der erste Todesfall in Deutschland durch Butangasinhalation zur Rauscherzeugung).

1997 starb ein 12-jähriger Junge in Nürnberg, nachdem er aus einer Nachfüllflasche Feuerzeuggas in einen (Luft-)Ballon gefüllt und daraus eingeatmet hatte.

1998 starb - neben dem bereits erwähnten Jugendlichen - ein 13-jähriger Junge in Marburg; dabei wurde berichtet, dass die Konsumenten das Gas auf ihre Kleidung verteilten, diese dann vor das Gesicht hielten und so konsumierten. Im gleichen Jahr verstarb ein 14-jähriger Junge in Kiel; er hatte in einem Kinderheim in eine Plastiktüte Feuerzeuggas aus einer Nachfüllflasche einströmen lassen und aus der Tüte inhaliert.

2001 starben nicht nur die beiden erwähnten Kinder in Gelsenkirchen und Karlsburg; in Frankfurt/Main starb ein 14-jähriger Junge nach dem Gasschnüffeln. In Marburg verstarb ein 14-jähriger Junge, nachdem er aus einer butangasgefüllten Plastiktüte inhaliert hatte. Kurze Zeit später verstarb in Kaufbeuren/Allgäu ein 15-jähriger Junge an den Folgen der Butangasinhalation.

In der MAK-Begründung [1] wird der Fall eines 15-jährigen Jungen berichtet, der nach Inhalation aus einem Butangas-Kanister eine teilweise reversible Hemiparese rechtsseitig aufwies. Dort wird auch die Intoxikation eines 16-jährigen Jungen aufgeführt, der nach einer nicht näher beschriebenen Butangasinhalation einen Herzstillstand erlitt.

In einer Arbeit aus der Universitätsklinik Innsbruck [2] wird der Fall einer akuten Butangasintoxikation eines 14-jährigen Jugendlichen mit letztendlicher Todesfolge nach $34 \mathrm{~h}$ geschildert. Er inhalierte Butangas aus einer Feuerzeugnachfüllflasche; zur Intensivierung zog er sich eine Plastiktüte über den Kopf. Als der Notarztwagen kam, wurde ein Kammerflimmern diagnostiziert. In der hämodynamischen Überwachung im Krankenhaus fielen eine Herzleistungsschwäche und eine Neigung zu vermehrten VES und SVES auf sowie weitere somatische Komplikationen. Neurologischerseits wurde der Verdacht auf ein hypoxisches Hirnödem gestellt; das CCT bestätigte die Diagnose eines massi- ven Hirnödems. Die durchgeführte transkranielle Doppler-Sonographie zeigte einen zerebralen Perfusionsstopp der Aa. carotides communes beidseits und des Circulus arteriosus Willisii.

Zur Erzeugung des Rauscherlebens ist zusammenfassend also eine erhebliche Konzentration des Butangases in der Einatemluft notwendig; eine genaue bzw. annähernde Konzentrationsangabe ist - naturgemäß - nicht möglich.

\section{Prävalenz}

Der zweckentfremdete Gebrauch von Lösungsmitteln und anderen potenziell Rausch erzeugenden Haushalts- und Industrieprodukten als „Schnüffelstoffe“ stellt ein internationales gesundheitspolitisches Problem von außerordentlicher Tragweite dar. Die Bezeichnung „schnüffeln“ leitet sich vom angloamerikanischen Wort „sniffing“ ab, das W. Ackerly und G. Gibson 1964 wählten, um das unersättliche Verlangen eines 8-jährigen mexikanischen Jungen nach Inhalation von Feuerzeugbenzin zu beschreiben [3].

Üblicherweise werden die Schnüffelstoffe in 3 (USA) bzw. 4 Gruppen eingeteilt:

- Gruppe der flüchtigen Lösungsmittel, z.B. Klebstoffe, Lacke, Verdünner

- Gruppe der Aerosole, darunter fallen insbesondere die Treibgase (In den USA werden die Aerosole unter die flüchtigen Lösungsmittel subsumiert.)

- Gruppe der Anästhetika, z.B. Chloroform, Lachgas, Ether

- Gruppe der flüchtigen Nitritverbindungen, z.B. Amylnitrit („Poppers“) [3]

Das als Thema anstehende Butangas wird nicht nur in Gaskartuschen zum Gebrauch als Brennmaterial verwendet, mit der internationalen Übereinkunft zur Verringerung der FCKW-haltigen Treibgase wurden Alternativen gesucht und Butan auch als Treibgas eingesetzt (z.B. in Haar- und Frischluftsprays). Insofern müsste Butan in die Gruppe der Aerosole (bzw. der flüchtigen Lösungsmittel) eingereiht werden.

Mitte der 60er-Jahre wurde erstmals in den USA bei Kindern und Jugendlichen der nordamerikanischen Großstädte und Ballungszentren der Gebrauch der Schnüffelstoffe beobachtet, jedoch lange Zeit als Kinderspiel bagatellisiert [4].

In England wurden die ersten Todesfälle durch Schnüffelstoffe 1971 berichtet; für die Mitte der 90er-Jahre wurde festgestellt, dass in England mehr Menschen an den Folgen des Missbrauchs von Schnüffelstoffen verstarben als an den Folgen der Heroinabhängigkeit [4].

1993 zeigte eine Studie der WHO, dass die Schnüffelstoffe die Hauptdroge der Straßenkinder weltweit sind; die meisten Straßenkinder leben in den Großstädten der sog. Entwicklungsländer. Das Ausmaß der körperlichen Schäden durch anhaltenden Gebrauch von Schnüffelstoffen übertrifft sogar die Auswirkungen des Alkoholismus. In einem Aktionsplakat der „national inhalant prevention coalition" werden die Schnüffelstoffe als "cocaine of the nineties“ bezeichnet [5]. 
Zur Verbreitung der Schnüffelstoffe - also nicht nur Butan - wird für die USA geschätzt, dass jeder 5 . Schüler in der 8 . Klasse bereits Konsumerfahrung hat. Die Schnüffelstoffe seien ebenso populär unter Jugendlichen wie Marihuana; mehr als 1000 käufliche Produkte seien als potenzielle Schnüffelstoffe anzusehen, alle seien legal zu erhalten. Als Einstiegsalter wird in den USA das 6.-16. Lebensjahr angegeben. Als besondere Gefahr aller Schnüffelstoffe wird immer wieder - auch in öffentlichen Kampagnen - das Sudden Sniffing Death Syndrome (plötzliches Schnüffeltod-Syndrom) betont.

In der Schweiz wurde 1998 eine Befragung unter 15-jährigen Schüler(inne)n durchgeführt bez. der Häufigkeit des Schnüffelns von Lösungsmitteln [6]. Bei den Antwortmöglichkeiten nie/einmal/mehrmals gaben an:

\begin{tabular}{llrlr}
\hline nie & Mädchen & $93,1 \%$ & Jungen & $92,5 \%$ \\
einmal & Mädchen & $3,9 \%$ & Jungen & $4,5 \%$ \\
mehrmals & Mädchen & $3,0 \%$ & Jungen & $3,0 \%$ \\
\hline
\end{tabular}

Im „Jahresbericht über den Stand der Drogenproblematik in der Europäischen Union 2001 “ wird aufgeführt, dass in Griechenland und Schweden der Konsum von Schnüffelstoffen genau so hoch bzw. größer sei als der von Cannabis [7].

Zur Frage der Verbreitung des Schnüffelstoffkonsums in Deutschland kann auf nur wenige Daten zurückgegriffen werden. Es entsteht fast der Eindruck, dass das Problem als solches nicht wahrgenommen wird. Diese Drogen werden bei der Betrachtung des Rauschmittelkonsums in der BRD so gut wie nicht erwähnt, Informationen sind nur sehr spärlich zu bekommen:

1. In der Repräsentativerhebung Drogen 1997 (Erwachsene) kommt die Gruppe „Schnüffelstoffe“ als eigenes Thema nicht vor, ebenso nicht in der von 2000. Allerdings finden sich Hinweise auf einen Gebrauch:

1997 wurde eine Lebenszeit-Prävalenz für Schnüffelstoffe von 0,7 gefunden für die Gesamtbevölkerung (Frauen 0,5/Männer 0,9), für die 18- bis 20-Jährigen eine von 1,6 und für die 21- bis 24-Jährigen eine von 2,4. Die Angaben bezogen sich nur auf Westdeutschland, in Ostdeutschland war die Prävalenz 0,0 [8].

2000 hat sich die Situation insofern geändert, als dass der Konsum in Westdeutschland ungefähr gleich geblieben ist (Lifetime-Prävalenz von 0,8), in Ostdeutschland schnellte jedoch die Lifetime-Prävalenz auf 0,6 hoch und glich sich damit ungefähr dem des Westniveaus an [9].

2. Auch in der Drogenaffinitätsstudie (Jugendliche) 1997 wird das Thema "Schnüffelstoffe“ nur nebenbei angeschnitten, ebenso in der von 2001.

1997 wird die Lifetime-Prävalenz ebenso wie die 12-MonatsPrävalenz der 12- bis 25 -jährigen Jugendlichen mit $1 \%$ angegeben, dabei zeigten sich für Ost- und Westdeutschland gleiche Ergebnisse. Allerdings ergaben sich Ost-West-Unterschiede beim Erstkonsum-Alter bei gleicher Geschlechterverteilung: Im Westen betrug das Erstkonsumalter 14,8 Jahre, im Osten $17,1[10]$.
2001 beträgt die Lifetime-Prävalenz der Schnüffelstoffe weiterhin 1\%, das Erstkonsumalter liegt bei 15,0 Jahren. Ein West-Ost-Unterschied scheint nicht mehr feststellbar (zum Vergleich: Das durchschnittliche Alter beim ersten Alkoholrausch lag 2001 bei 15,6 Jahren) [11].

(Zu den Prävalenzzahlen merken beide zuletzt erschienenen Lehrbücher der Suchtmedizin [3,12] an, dass diese aufgrund methodischer Probleme der Erhebung von Daten bei Kindern und Jugendlichen nicht deren realen Gebrauch widerspiegeln.)

3. Im „nationalen Rauschgiftbekämpfungsplan“ von 1990 [13] stehen keine Hinweise auf den Gebrauch von „Schnüffelstoffen“, ebenso nicht im „Landesprogramm gegen Sucht“ [14] von 1999 des Landes NRW.

4. Im Drogen- und Suchtbericht vom Mai 2002 der Drogenbeauftragten der Bundesregierung [15] werden die Schnüffelstoffe ebenso wenig erwähnt wie im sog. Drogenaktionsplan des Bundesministeriums für Gesundheit „Vorbeugen.Verhindern. Helfen. Neue Konzepte der Drogen- und Suchtpolitik“ [16].

5. Im Jahrbuch Sucht 2002 (DHS) werden die Schnüffelstoffe nicht erwähnt [17].

6. Das Bundeskriminalamt führt keine eigene Statistik zur Frage der Toten durch Schnüffelstoffe oder speziell Butangas im Rahmen von Rauscherleben.

7. Nur aus dem Internet ist zu entnehmen, dass es in Deutschland wahrscheinlich (erst) 1996 zum ersten Todesfall durch Butangasinhalation gekommen ist [18].

8. Zu Todesfällen durch andere Schnüffelstoffe sind kaum Aussagen zu finden; in einem Artikel der Schweriner Volkszeitung vom 6.1.2001 wird der Tod eines 14-jährigen Jungen aus Mecklenburg im Herbst 2000 berichtet, der am Konsum eines nicht genannten Schnüffelstoffes gestorben war. In diesem Artikel steht der Hinweis, dass es jährlich zu 30-50 Todesfälle durch Schnüffelstoffe komme [19].

Als Beispiel für die Verbreitung des Schnüffelstoffkonsums kann das Setting einer Kinder- und Jugendpsychiatrie, die eine spezielle Suchtstation vorhält, geschildert werden: Alle Feuerzeuge, Haarsprays etc. sind auf der Station unter Verschluss; die Erfahrung hatte gezeigt, dass alle Patient(inn)en geschnüffelt hatten auch auf Station. Auch bei den Fachstellen für Suchtprävention NRW ist dieses Phänomen bekannt: Auf den Koordinationstreffen der Fachkräfte wurde in den letzten drei Jahren wiederholt nach Informationen zum Wirkmechanismus der Gasinhalation gefragt, da regelmäßig Anfragen von Schulen, Jugendzentren und Heimeinrichtungen an die Fachstellen herangetragen werden.

Im Folgenden wird auf der Basis empirischer Beobachtungen und bekannter medizinisch-naturwissenschaftlicher Daten ausschließlich auf den inhalativen Konsum von Butangas eingegangen.

\section{Das Gas und seine Wirkung}

Butan ist ein Bestandteil des Erdgases; es gehört chemisch gesehen zu den Alkanen. 
Es ist farb- und geruchlos, schwerer als Luft, schlecht löslich in Wasser, aber gut löslich in Benzin und ähnlichen Lösungsmitteln und es ist brennbar.

Im Handel wird Butangas unter die Flüssiggase subsumiert. Unter Flüssiggasen versteht man C3- und C4-Kohlenwasserstoffe bzw. Gemische daraus, wie sie in der Natur vorkommen oder als Synthesegas anfallen. Im Sinne der technischen Regeln Flüssiggas (und des DVGW-Arbeitsblattes G 260) werden unter Flüssiggas Propan, Butan und Propan-Butan-Gemische verstanden. Flüssiggase werden in grauen oder roten Flüssiggasflaschen geliefert, die durch ihr Vorkommen beim Camping, im Caravan und in anderen Bereichen von Haus, Garten und Hobby allgemein bekannt sind.

Das Flüssiggas entsteht bei der Rohölverarbeitung in den Raffinerien, ist aber auch Begleitgas bei der Förderung von Erdgas und Erdöl.

Aus der MAK-Begründung [1], die die wesentlichen toxikologischen und arbeitsmedizinischen Fakten (Stand 1999) aufführt, ist zusammengefasst zu entnehmen, dass die Exposition gegenüber hohen Butangaskonzentrationen eine zentralnervöse Depression (im MAK-Text wohl als Funktionsminderung gemeint) bis hin zur Narkose bewirkt. Auch erhöht Butangasinhalation die Empfindlichkeit des Herzmuskels gegenüber Adrenalin; der genaue Mechanismus sei nicht geklärt. (Diese Konstellation wird als Pathomechanismus für das Sudden Sniffing Death Syndrome diskutiert.) Als psychische Wirkung wurde von 12 Personen, die Butangas zur Rauscherzeugung inhaliert hatten, das Auftreten von Euphorie und Halluzinationen beschrieben.

Neuere und weitergehendere Informationen zum Thema (Stand 1.11.00) finden sich in der Stoffdatenbank „Gefahrstoffinformationssystem der gewerblichen Berufsgenossenschaften (GESTIS)“, abzurufen über das Berufsgenossenschaftliche Institut für $\mathrm{Ar}$ beitssicherheit (BIA). Dort wird unter den „Hauptwirkungsweisen/akut" aufgeführt:

- „Erfrierungen bei Hautkontakt mit der unterkühlten Flüssigkeit

- narkotisierende Wirkung hoher Inhalationskonzentrationen

- Tod durch Ersticken bei Sauerstoffverdrängung“ [20]

\section{Die Butangasinhalation und ihre Folgen}

Die Inhalation von Butangas verändert die Zusammensetzung der normalerweise vorhandenen Einatemluft: Durch das Butangas werden die Volumenanteile der normalen Bestandteile verkleinert.

In der Nähe der Erdoberfläche besteht die Erdatmosphäre zu ca. 78 Vol.-\% aus Stickstoff, zu ca. 21 Vol.-\% aus Sauerstoff (entspricht einem Sauerstoffpartialdruck von ca. $150 \mathrm{~mm} \mathrm{Hg}$ ), der verbleibende Rest von ca. 1 Vol.-\% setzt sich zusammen aus anderen Gasen wie Argon, Kohlendioxid etc. Aufgrund der Gegebenheiten des Respirationstraktes (Anfeuchtung der Einatemluft bis zur vollständigen Wasserdampfsättigung und damit Vol.\%-Verschiebung, Durchmischung der in den Lungen im Totraum und im Residualvolumen verbleibenden Luft mit der Einatem- luft) sinkt die Sauerstoffkonzentration in den Alveolen auf ca. 14 Vol.-\% (entspricht einem Sauerstoffpartialdruck von ca. $100 \mathrm{~mm}$ $\mathrm{Hg}$ ) ab. Dieser Sauerstoffanteil steht dem Organismus zum Gasaustausch zur Verfügung. Die treibende Kraft für den Gasaustausch ist das Partialdruckgefälle des Sauerstoffpartialdruckes in den Alveolen einerseits (ca. $100 \mathrm{~mm} \mathrm{Hg}$ ) und des Sauerstoffpartialdruckes des venösen Blutes andererseits (ca. $40 \mathrm{~mm} \mathrm{Hg}$ ) $[21,22]$.

Zur Darstellung des Pathomechanismus ist es sinnvoll, ausgehend von pathophysiologischen Daten, das Absinken des Sauerstoffpartialdrucks im arteriellen Blut, in der Medizin Hypoxämie genannt, zu betrachten im Hinblick auf die entstehende Symptomatik und dann auf die Zusammensetzung der Einatemluft rückzurechnen. Dabei wird vorausgesetzt, dass der Körper nicht durch Krankheiten, die die Atmung, den Gasaustausch, den Sauerstofftransport o.Ä. betreffen, geschädigt ist. Ferner wird vereinfachend angenommen, dass das organisch bedingte „normale“ Verhältnis Sauerstoffkonzentration in der Einatemluft : Sauerstoffkonzentration in den Alveolen (s.o.: 150 : 100, also 3 : 2) konstant bleibt. Dieses Vorgehen ist erforderlich, da sich menschliche Experimente im Hinblick auf die möglichen Schädigungen verbieten.

Die arterielle Hypoxämie wird eingeteilt in unterschiedliche Schweregrade je nach dem vorhandenen Sauerstoffpartialdruck im arteriellen Blut: Als leichte Hypoxämie wird bezeichnet ein arterieller Sauerstoffpartialdruck von $69-62 \mathrm{~mm} \mathrm{Hg}$, als mittelschwere einer von 61-51 mm Hg, als schwere (= dringend therapiebedürftig) einer von 50-40 mm Hg und als eine sehr schwere (= lebensbedrohliche) einer von unter $40 \mathrm{~mm} \mathrm{Hg}$.

Von den genannten Daten ausgehend müsste also überschlagsmäßig ein Sauerstoffpartialdruck von ca. 40 mm Hg in den Alveolen herrschen, damit eine Sauerstoffminimalversorgung im Rahmen einer sehr schweren Hypoxämie im Organismus aufrechterhalten werden kann. Das entspräche einem Sauerstoffanteil von ca. 5-6 Vol.-\% in den Alveolen; rückgerechnet wären ca. 7,5-9 Vol.-\% Sauerstoffanteil in der Einatemluft notwendig. Um ein Absinken des Sauerstoffangebotes in der Einatemluft auf 7,5-9 Vol.-\% - und korrespondierend das Absinken des Stickstoffanteiles auf ca. 28-33,5 Vol.-\% - zu erreichen, ist eine Butangaskonzentration von ca. 60-65 Vol.-\% notwendig. Mit anderen Worten: Bei einer Butangaskonzentration von mehr als ca. 60-65 Vol.-\% in der Einatemluft ist die Funktion des Organismus massiv gefährdet.

Für die leichte Hypoxämie würde gelten: Um den Sauerstoffpartialdruck von $69 \mathrm{~mm} \mathrm{Hg}$ im Blut zu erreichen, muss das alveolare Sauerstoffangebot ca. $70 \mathrm{~mm} \mathrm{Hg}$ bzw. entsprechend ca. 10 Vol.-\% Sauerstoff betragen, in der Einatemluft wären also ca. 15 Vol.-\% Sauerstoff notwendig. Korrespondierend würde der Anteil von Stickstoff in der Einatemluft ca. 56 Vol.-\% betragen. Mit anderen Worten: Ab einer Butangaskonzentration von ca. 30 Vol.-\% in der Einatemluft muss mit dem Auftreten einer Hypoxämie gerechnet werden. 
Letztendlich ergibt sich daraus, dass der Korridor zur Wirksamkeit der Inhalation einerseits und dem Auftreten erster Schäden andererseits mit 30-60/65 Vol.-\% Butangas in der Einatemluft sehr gering ist.

Aus den eingangs geschilderten Konsummechanismen kann berechtigt vermutet werden, dass bei der Butangasinhalation zum Zwecke der Rauscherzeugung die Konzentration von 30 Vol.-\% Butangas in der Einatemluft mit an Sicherheit grenzender Wahrscheinlichkeit überschritten wird. Eine Butangaskonzentration von mehr als 60 Vol.-\% in der Einatemluft ist unter den geschilderten Umständen nicht auszuschließen.

\section{Ablauf der Butangasinhalation unter pathophysiologischen Aspekten}

Der Gebrauch von Butangas zur Rauscherzeugung wird in der Suchtmedizin fast ausnahmslos betrachtet im Rahmen der zusammenfassenden Gruppe „Schnüffelstoffe“. Dabei wird festgestellt, dass die Gruppe chemisch sehr heterogen sei, aber eine vergleichbare Anwendung („schnüffeln“) und Erlebnisqualität habe $[3,12]$. Insofern ist diesen Abhandlungen nicht viel Themenspezifisches zu entnehmen.

Zwei unterschiedliche Ansätze zur Pathophysiologie der Akutfolgen der Butangasinhalation liegen in einem Skript der Universität Göttingen [23] vor; dort steht der Satz: „Mediziner erklären die Todesfälle folgendermaßen: Das Butan kann sich mit dem Hämoglobin des Blutes verbinden und blockiert so die roten Blutkörperchen." Ein Beleg für diesen Ansatz wird nicht genannt, insbesondere wird nicht erwähnt, ob die Affinität des Butangases gegenüber Hämoglobin größer ist als die des Sauerstoffs oder ob das Butangas nur dann gebunden wird, wenn kaum noch bzw. gar kein Sauerstoff mehr zur Verfügung steht. Aus den in der MAK-Begründung aufgeführten Ergebnissen kann berechtigt rückgeschlossen werden, dass die Affinität von Butan gegenüber dem Hämoglobin kleiner als die von Sauerstoff ist, es hätte sich sonst in den Langzeitversuchen anreichern müssen. Im Skript wird dann die Frage gestellt: „Wäre es aber nicht auch denkbar, dass das relativ schwere Gas Butan sich in den Lungen der Jugendlichen befand und sie aufgrund seiner Dichte nicht mehr durch die Luftröhre verlassen konnte?" Auch dieses Argument, dass aufgrund der Schwere des Gases die Alveolen „verstopfen“, hätte in den Langzeitversuchen zu entsprechenden Konsequenzen führen müssen. Da diese nicht beobachtbar waren, entfällt auch dieses Argument.

Zurückgreifend auf die MAK-Begründung [1] ist festzustellen, dass mehr oder weniger keine der dort aufgeführten Inhalationsmengen zu Störungen führte. Auch eine länger andauernde Exposition gegenüber $500 \mathrm{ml}$ Butangas $/ \mathrm{m}^{3}$ rief keine butangasassoziierten Störungen hervor. Zusammengefasst können daher keine Butangas-spezifischen Akutfolgen einer Inhalation in den dort angegebenen Konzentrationen festgestellt werden.

Resümierend entsteht aus den toxikologischen und arbeitsmedizinischen Fakten (s.o.) eher das Bild eines in sich nicht hochtoxischen Gases, dessen akut gesundheitsschädliche Wirkungen im Bereich der Rauscherzeugung am wahrscheinlichsten auf den pathophysiologischen Konsequenzen der akuten Verdrängung des Sauerstoffes in der Einatemluft durch das Gas und der daraus resultierenden Sauerstoffmangelversorgung beruhen. Dieser Wirkmechanismus wird in der GESTIS-Stoffdatenbank [20] auch klar benannt. Je nach Intensität der Inhalation, also der Konzentration des Gases in der Einatemluft und damit der Reduktion des Sauerstoffangebotes, kann es bis zur vollständigen Verdrängung des Sauerstoffes durch das Gas kommen. Diese Konstellation wird üblicherweise als Asphyxie bezeichnet.

Das Wort Asphyxie kommt aus dem Griechischen und heißt wörtlich übersetzt „Pulslosigkeit“. Es wird in der Medizin verwendet zur Beschreibung einer Atemdepression bzw. eines Atemstillstandes infolge Herz-Kreislauf-Versagen, Atemwegsverlagerung oder Atemlähmung; die Asphyxie führt zur partiellen oder evtl. sogar zur kompletten Gasaustauschbehinderung zwischen Zelle und Umwelt. Je nach Lokalisation der Gasaustauschbehinderung kann differenziert werden zwischen einer inneren (z.B. Atemenzymschädigung durch Blausäure) und einer äußeren Erstickung (Ertrinken, Erdrosseln, Sauerstoffentzug u.a.m.). Vom Asphyxiebeginn bis zur irreversiblen Zellschädigung vergeht eine von der Sauerstoffmangelempfindlichkeit der einzelnen Gewebe abhängige Zeitspanne, die bei den Hirn- und Herzmuskelzellen mit etwa 4 min am Geringsten ist [24].

Im konkreten Fall der Butangasintoxikation stellt sich mit zunehmender Gas-Inhalation und daraus resultierendem Sauerstoffmangel zunächst eine Hypoxie ein, die der Organismus durch Intensivierung der Atmung/Hyperventilation zu kompensieren versucht.

Die klinische Zeichen der Hypoxie sind:

- Tachykardie und Blutdruckanstieg

- Steigerung der Atmung

- Zyanose

- Schwitzen

- Unruhe, Erregtheit, Verwirrtheit

- Bewusstseinseintrübung über Schläfrigkeit, Somnolenz bis hin zur Bewusstlosigkeit.

Falls die Kompensationsmaßnahmen des Organismus nicht ausreichen, kommt es zum Stadium der Dekompensation mit den klinischen Zeichen:

- Blutdruckabfall

- Bradykardie, Herzrhythmusstörungen, Herzstillstand.

Aus der kompensatorischen Hyperventilation resultiert eine Hypokapnie, die ihrerseits im Regelkreis zur Sicherstellung der Hirndurchblutung zu einer Kontraktion der Hirngefäße und zu einer respiratorischen Alkalose mit Verminderung des Atemreizes führt, also die Sauerstoffversorgung des Gehirns eher weiter verschlechtert. (Memo: Der stärkste Atemreiz ist nicht der Sauerstoffmangel, sondern eine akute Hyperkapnie.)

\section{Sauerstoffmangel im Gehirn}

Das ZNS ist gegenüber Sauerstoff- (und Glukose-)Mangel sehr empfindlich. 
Obwohl das Gehirn nur 2\% des Körpergewichtes ausmacht, braucht es ca. 15\% des Herzminutenvolumens, das sind ca. 750-1000 ml Blut/min; etwa 20\% des Ruheenergiebedarfes eines Menschen entfallen auf das Gehirn. Den Energiebedarf deckt das Gehirn fast ausschließlich durch den oxidativen Abbau von Glukose. Pro Minute sind ca. $50 \mathrm{ml}$ Sauerstoff und $80 \mathrm{mg}$ Glukose zur Versorgung des Gehirns notwendig, dabei wird die Großhirnrinde etwa fünfmal stärker durchblutet als die Marksubstanz, die graue Substanz verbraucht etwa fünfmal mehr Sauerstoff als die weiße. Die Nervenzellen selber reagieren also am empfindlichsten auf Sauerstoffmangel, daher wird die graue Substanz eher geschädigt als die übrigen Hirnstrukturen [25].

Die Unterbrechung der Sauerstoffzufuhr zum Gehirn führt zu einem raschen Erlöschen von dessen Funktion: Nach 6-8 Sekunden findet sich kein molekularer Sauerstoff mehr in der grauen Substanz, EEG-Veränderungen treten auf, nach 10-12 s kommt es zur Bewusstlosigkeit. Bei einer Unterbrechung der Hirndurchblutung länger als 5 min muss mit dem Auftreten erster irreversibler Schäden gerechnet werden: Es kommt zu einer Anreicherung von Laktat - das per se bereits durch Ansäuerung Hirngewebsnekrosen hervorrufen kann - durch Änderung der Glykolyse und von Adenosin sowie zur Störung im Protein- und Lipidstoffwechsel. Der Zusammenbruch des Energiestoffwechsels führt seinerseits zu einem Hirnödem [26].

\section{Sauerstoffmangel am Herzen}

Die Funktionselemente des Herzens sind die Herzmuskelfasern; morphologisch und funktionell können zwei Typen von Herzmuskelfasern unterschieden werden: die Arbeitsmuskulatur (Myokard) und das spezifische Erregungsbildungs- und -leitungssystem.

Die besondere Vulnerabilität des Myokards in Bezug auf Sauerstoffmangel erklärt sich aus der Eigenart des Herzmuskelgewebes, das seine Energie überwiegend aus dem oxidativen Abbau von Nährstoffen bezieht und daher eine sog. Sauerstoffschuld durch anaerobe Prozesse nicht eingehen kann. Die Verminderung des Sauerstoffangebotes im Blut bewirkt aus diesem Grund am Herzen eine Abnahme der myokardialen Kontraktilität, die letztendlich zur Dilatation führt. 6-10 min nach Unterbrechung der Sauerstoffzufuhr endet die Pumpfunktion und das Herz steht still.

Im Bereich des spezifischen Erregungsbildungs- und -leitungssystems kann Sauerstoffmangel Extrasystolen (VES) provozieren; je schlechter die Sauerstoffversorgung des Herzens ist, um so mehr VES treten auf. Ereignen sich diese in der Erregungsrückbildungsphase als frühzeitig einfallende VES (sog. R-auf-T-Phänomen), kann es zur ventrikulären Tachykardie (Kammerflimmern) kommen. Insofern kommt der Ischämie eine Triggerfunktion beim plötzlichen Herztod zu [27, 28].

Daher ist zusammenfassend festzuhalten, dass die Asphyxie selber, also die Behinderung des Gasaustausches in diesem Fall durch Fehlen von Sauerstoff, innerhalb von 5-10 min zum asphyktischen Kreislaufstillstand (Asystolie) führen kann.
Die User

Bereits in der Literatur wird mehrfach beschrieben, dass die Butangasinhalation zu Euphorie und Halluzinationen führt; die Schilderung der von der Polizei befragten Jugendlichen war noch spezifischer: Sie berichteten von sexuellen Gefühlserlebnissen.

Auf dem Hintergrund des wahrscheinlichen Pathomechanismus der zerebralen Hypoxie einerseits und der Erlebnisqualität andererseits ist zu fragen, ob „angenehme Gefühle“ durch Hypoxie hervorgerufen werden können; mehr oder weniger jeder Medizinstudent wird in der Rechtsmedizin mit Opfern autoerotischer Unfälle konfrontiert und ist daher zur Thematik „sexuelle Stimulation durch Hypoxie“ gut informiert.

Sehr detailliert werden die autoerotischen Stimulationsmechanismen durch Erzeugung einer zerebralen Hypoxie im sadomasochistischen Schrifttum dargestellt, die jedem über das Internet offen zugänglich sind [29]. Hierin wird auch die Verwendung von Butangas erwähnt, jedoch von dieser Praxis wegen der gesundheitlichen Risiken abgeraten. Zur Motivation der autoerotischen Stimulation durch Hypoxie finden sich dort ausreichende Hinweise.

Unter den Usern der Schnüffelstoffe allgemein lassen sich vom Konsumentenprofil her drei Gruppen definieren:

- die lösungsmittelabhängigen Erwachsenen; sie sind in aller Regel schwerst beeinträchtigte chronisch kranke Abhängige mit einem Altersgipfel von 25-30 Jahren,

- die polytoxikomanen Jugendlichen; sie konsumieren alles, von dem sie sich eine psychotrope Wirkung versprechen, Altersgipfel 16-18 Jahre,

- die schnüffelnden Kinder.

Vom Konsumverhalten her können ebenfalls drei Gruppen differenziert werden:

- die Probierer/Experimentierer, Alter 10-14 Jahre, aber auch jünger; befriedigen ihre Neugier und stellen nach einigen Versuchen den Konsum ein.

- die sozialen Gruppen- oder Freizeitmissbraucher; das Schnüffeln wird als mehr oder weniger regelmäßiges Ritual zum Gruppenzusammenhalt gebraucht.

- die chronischen Einzelschnüffler; beginnen meist in der Gruppe, dann jedoch bekommt das Rauscherleben die Priorität vor dem Gruppenerleben und sie konsumieren häufiger und intensiver als die übrigen Mitglieder der Gruppe.

Zur Motivation von Kindern und Jugendlichen, im Rahmen risikobehafteten Probierverhaltens unterschiedlichste Sorten von Rauschmitteln zu konsumieren, gibt es im Bereich der Prävention ausreichend Schrifttum [30]: Ein wichtiger Faktor ist die Neugier, die aber nicht zur Chronifizierung des Schnüffelns führt. Ein weiterer ist die Modulation der Affektivität durch das eingesetzte Rauschmittel: Wenn das Lebensgefühl ist, „zur falschen Zeit im falschen Film auf dem falschen Stern in der falschen Galaxie“ zu sein, liegt es nahe, einen Weg zu suchen, der dieses Gefühl aushaltbar macht. Dies kann einfach und zuverlässig durch Rauschmittel geschehen. 
Es stellt sich dann noch die „praktische“ Frage, warum die Butangasinhalation zur Rauscherzeugung von Kindern und Jugendlichen genutzt wird. Der Vorteil des Butangases liegt in der einfachen Handhabbarkeit und Verfügbarkeit auch für Kinder und Jugendliche:

- Es ist legal und überall erhältlich, die Kosten sind minimal, die Gasbehälter sind klein und jederzeit mit sich zu führen, daher ist das Rauscherleben in fast jeder Situation möglich.

- Beim „Erwischtwerden“ ist es fast unverdächtig - weil die meisten Jugendlichen rauchen -, auch ist Butan nicht in den derzeitigen Drogentesten nachweisbar.

- Der Konsum kann mehr oder weniger jederzeit erfolgen; ein Aufschub des Konsums ist nicht notwendig.

- Und: Schnüffelstoffe werden nicht über Dealer vertrieben, d.h., die Kinder und Jugendlichen brauchen keine Beziehungen zum Erwerb: Sie sind nicht abhängig von einem Stoff-Lieferanten.

Es gibt noch eine spezielle Situation, in der die Schnüffelstoffe nicht unwichtig zu sein scheinen: in der stationären Therapie. Dort können natürlich keine nachweisbaren Drogen konsumiert werden [31]. In diesem durch Kontrolle geprägten Klima sind Schnüffelstoffe eine Alternative als Ersatzdroge.

\section{Stoffe mit vergleichbarem Wirkmechanismus}

$\mathrm{Zu}$ fragen ist, ob es unter den Schnüffelstoffen Substanzen gibt, die einen vergleichbaren Wirkmechanismus haben, also das aktuelle Rauscherleben nicht durch ihre chemische Eigenart hervorrufen, sondern durch den Mechanismus der Sauerstoffverdrängung.

Aus den toxikologisch-medizinischen Fakten zum Lachgas (chem. Summenformel: $\mathrm{N}_{2} \mathrm{O}_{1}$ ) ist zu entnehmen, dass dieses Gas eine berauschende Wirkung besitzt [32]. Es wird im medizinischen Kontext zur Narkose eingesetzt. Zur Narkose wird ein Gemisch aus 25\% Sauerstoff und 75\% Lachgas benutzt; nur bei dieser hohen Sauerstoffkonzentration ist eine gute Verträglichkeit gegeben.

Im alltäglichen Gebrauch ist es bekannt aus den Gaskartuschen der Sahnesyphons.

Als Rauschmittel wird es z.B. abgegeben in „Luft“-Ballons, die statt mit Luft mit Lachgas gefüllt sind. Die Inhalation erfolgt durch Einatmung aus dem Ballon; dabei sind natürlich keine 25\% Sauerstoff vorhanden. Damit gelten die für Butangas und die Hypoxie erarbeiteten Gefahren. Die Nachfrage nach Lachgas ist so groß, dass Vorräte gezielt gestohlen werden [33].

Sowohl im Internet [34] als auch in einer Szene-Zeitung [35] wird der Konsum von Lachgas als gesundheitlich unbedenklich dargestellt. Diese Einschätzung muss auf der Basis der vorgenannten Überlegungen zum pathophysiologischen Wirkmechanismus von Rauscherleben und Gefahren auch für dieses Gas als Desaster bezeichnet werden. Die Zielgruppe der Konsumenten besitzt keinen Zugang zu den Informationen bezüglich der Gefahren, die meisten Mediziner leider ebenso nicht. Hier will der Aufsatz eine Wissenslücke schließen.
Lachgas nimmt im Rahmen der Schnüffelstoffe insofern eine Sonderstellung ein, als dass der unsachgemäße Gebrauch von Lachgas, also zum Konsum als Rauschmittel, einen Verstoß gegen das Arzneimittelgesetz darstellt [36].

\section{Ergebnis}

Die Aufgabenstellung des Aufsatzes bestand in der Beschreibung eines das Rauscherleben und die Todesfälle erklärenden gemeinsamen Pathomechanismus; dieser beruht auf der Hypoxie infolge Sauerstoffverdrängung durch Butangas.

Dabei ist die „therapeutische Breite“ bezüglich der Konzentration des Butangases sehr genau definiert: Unter ca. 30 Vol.-\% Butangas in der Einatemluft ist keine Wirkung erwartbar, bei über ca. 60-65 Vol.-\% ist das Auftreten lebensgefährlicher Zwischenfälle sehr wahrscheinlich.

Die Verwendung von Butangas zur Rauscherzeugung ist zusammenfassend am ehesten im Bereich der autoerotischen Stimulation durch Erzeugung einer Hypoxie anzusiedeln; insofern wären die Todesfälle als autoerotische Unfälle zu klassifizieren.

Diese Einschätzung korreliert mit dem Auffindungsmodus des Jugendlichen 1998, wobei berechtigt vermutet werden kann, dass das minimal notwendige Sauerstoffangebot für längere Zeit deutlich unterschritten wurde. Auch der in der MAK-Begründung aufgeführte Fall mit direkter Inhalation aus einem Butangas-Kanister lässt eine massive Butangaskonzentration vermuten und stützt daher die Arbeitshypothese.

Letztendlich ist im Fallbericht des 14-jährigen Jugendlichen aus Innsbruck genau die Symptomatik bezüglich Herz und Gehirn beschrieben, die nach den Überlegungen zur Pathophysiologie der systemischen Hypoxie zu erwarten war. Insofern stellt dieser Fallbericht eine Art Verifizierung der Arbeitshypothese dar.

Den Kindern und Jugendlichen ist ein autoerotischer Unfall mit Todesfolge bekannt durch den Tod von Michael Hutchence (12.1.1960-22.11.1997), einem (ehemaligen) Mitglied der australischen Gruppe INXS.

\section{Epilog: Folgerungen}

Auch wenn in der Drogenaffinitätsstudie von 2001 keine eigengewichteten Aussagen zum Gebrauch von Schnüffelstoffen unter Jugendlichen in der BRD zu finden sind (s.o.), zeigt sich im „Anhang“ der Studie eine wichtige Tendenz: Bei drogenunerfahrenen Jugendlichen nimmt die generelle Ablehnung des Drogenkonsums kontinuierlich seit 1989 (erste verfügbare Daten) ab, die Akzeptanz vom Cannabis-Konsum nimmt deutlich zu, zumindest als Probierverhalten. Auch das Probierverhalten gegenüber anderen Drogen [37] scheint zuzunehmen.

Daher muss berechtigt befürchtet werden, dass das Probierverhalten von Kindern und Jugendlichen nicht vor den Schnüffelstoffen Halt machen wird. Aufgrund des Alters der Erstkonsumenten von Schnüffelstoffen kann sogar die These gewagt 
werden, dass die Schnüffelstoffe - neben oder evtl. sogar vor Alkohol und Nikotin - als Einsteigerdrogen für konsumierende Kinder und Jugendliche von immens wichtiger Bedeutung sind [38].

Die katastrophalen medizinischen Auswirkungen des andauernden Konsums von Schnüffelstoffen - nicht nur von Butan - einerseits, ihre Funktion als Einstiegsdroge andererseits sowie die potenziell tödlichen Folgen bereits des Erstkonsums würden ein „Umdenken“ in der drogenpolitischen Diskussion hin zur Wahrnehmung des Schnüffelstoff-Problems sinnvoll erscheinen lassen.

\section{Literatur}

${ }^{1}$ Greim H (Hrsg). Gesundheitsschädliche Arbeitsstoffe. Toxikologischarbeitsmedizinische Begründungen von MAK-Werten (Maximale Arbeitsplatzkonzentrationen) [...] der Senatskommission zur Prüfung gesundheitsschädlicher Arbeitsstoffe der Deutschen Forschungsgemeinschaft, n- und iso-Butan, 1-9, 29 Lieferung. Weinheim: WileyVCH Verlag GmbH 1999

${ }^{2}$ Rieder-Scharinger J, Peer R, Rabl W et al. Multiorganversagen nach Butangasinhalation: Ein Fallbericht. Wien Klin Wochenschr 2000; 112 (24): $1049-1052$

${ }^{3}$ Thomasius R. Flüchtige Lösungsmittel. In: Gölz J (Hrsg) Moderne Suchtmedizin. B 4.10-1-4.10-6, ebendort: Klinischer Verlauf der Abhängigkeit von flüchtigen Substanzen, B 7.2.9.-1f, ebendort: Folgeerkrankungen der Schnüffelstoffe, C 4.3-1-4.3-6. Stuttgart: Thieme 1999

${ }^{4}$ Thomasius R. Schnüffelstoffe In: DHS (Hrsg) Jahrbuch Sucht 1996. Geesthacht: Neuland Verlagsgesellschaft mbH 1995; 178-190

${ }^{5} \mathrm{http}$ //www.inhalants.org. USA: National inhalant prevention coalition Abrufdatum: 7.11.2002

${ }^{6}$ Schweizerische Fachstelle für Alkohol- und andere Drogenprobleme (Hrsg). drogeninfo schnüffeln. Lausanne (siehe auch: www.sfaispa.ch)

${ }^{7}$ Europäische Beobachtungsstelle für Drogen und Drogensucht (Hrsg). Jahresbericht über den Stand der Drogenproblematik in der Europäischen Union 2001, (über Internet http://www.emcdda.org/infopont/ publications/annrep.shtml). Luxemburg 2001

8 Kraus L, Bauernfeind R. Repräsentativerhebung 1997. Schriftliche Befragung zum Gebrauch psychoaktiver Substanzen bei Erwachsenen in Deutschland, erstellt im Auftrag des Bundesministeriums für Gesundheit, Referat für Presse- und Öffentlichkeitsarbeit. Bonn 1997

${ }^{9}$ Kraus L, Augustin R. Repräsentativerhebung zum Gebrauch psychoaktiver Substanzen bei Erwachsenen in Deutschland 2000. Sucht 2001; Sonderheft 1

${ }^{10}$ Bundeszentrale für gesundheitliche Aufklärung. Die Drogenaffinität Jugendlicher in der Bundesrepublik Deutschland 1997. Köln 1998

${ }^{11}$ Bundeszentrale für gesundheitliche Aufklärung. Die Drogenaffinität Jugendlicher in der Bundesrepublik Deutschland 2001. Köln 2001

12 Schulze-Alexandru M, Kovar KA. Schnüffelstoffe In: Uchtenhagen A, Zieglgansberger (Hrsg). München 2000; 117-126

${ }^{13}$ Der Bundesminister für Gesundheit, der Bundesminister des Inneren (Hrsg). Nationaler Rauschgiftbekämpfungsplan. Maßnahmen der Rauschgiftbekämpfung und der Hilfe für Gefährdete und Abhängige. Bonn 1990

${ }^{14}$ Ministerium für Frauen, Jugend, Familie und Gesundheit des Landes Nordrhein-Westfalen (Hrsg). NRW-Landesprogramm gegen Sucht. Eine Gemeinschaftsinitiative. Düsseldorf 1999
${ }^{15}$ Die Drogenbeauftragte der Bundesregierung. Drogen- und Suchtbericht. Bonn 2002

${ }^{16}$ Bundesministerium für Gesundheit. Vorbeugen. Verhindern. Helfen. Neue Konzepte der Drogen- und Suchtpolitik. Bonn 2002

${ }^{17}$ DHS (Deutsche Hauptstelle gegen die Suchtgefahren) (Hrsg). Jahrbuch Sucht 2002. Geesthacht: Neuland Verlagsgesellschaft mbH 2001

${ }^{18}$ Scheuelbergschule Bargan, Grund- und Hauptschule mit Werkrealschule in Schwäbisch-Gmünd. Info: Legale Drogen, siehe: www.sbs.aa.schule-bw.de/legal.htm

${ }^{19}$ Schweriner Volkszeitung vom 6.1.2001. Der Tod aus der Tüte. Wenn Spray und Klebstoff zur Ersatzdroge werden (über Internet: www.svz.de/archiv.newsdw/2001/Januar/06./Vermischtes/tuete.html)

${ }^{20}$ Berufsgenossenschaftliche Institut für Arbeitssicherheit (BIA). Gefahrstoffinformationssystem der gewerblichen Berufsgenossenschaften (GESTIS): www.hvbg.de/d/bia/fac/zesp/zesp.htm

${ }^{21}$ Siegenthaler W (Hrsg). Klinische Pathophysiologie. Stuttgart: Thieme 1994

${ }^{22}$ Schmidt RF, Thews G (Hrsg). Physiologie des Menschen. Berlin: Springer 1980

${ }^{23}$ Universität Göttingen. www.stud.uni-goettingen.de/ s193271/organikum/aufg1.htm, (Zitate S. 2, Nr. 13.).

${ }^{24}$ Riede UN, Schaefer HE (Hrsg). Allgemeine und spezielle Pathologie. Stuttgart: Thieme 1995

${ }^{25}$ Blümcke S. Pathologie. Berlin: Springer 1995

${ }^{26}$ Lasch HG, Lenz K, Seeger W. Lehrbuch der internistischen Intensivtherapie. Stuttgart: Thieme 1997

${ }^{27}$ Brisse B, Bender F (Hrsg). Autonome Innervation des Herzens. Myokardiale Hypoxie. Darmstadt: Steinkopf Verlag 1987

28 Blaeser-Kiel G. Plötzlicher Herztod. Dem elektrophysiologischen Unfall auf der Spur. Deut Ärzteblatt 2002; 99: B $941 \mathrm{f}$

${ }^{29}$ Datenschlag. „deutschsprachige Gruppe, die Informationen über sadomasochistische Themen zusammenträgt und aufbereitet" www.datenschlag.org.

${ }^{30}$ Ministerium für Arbeit, Gesundheit und Soziales. Sucht hat immer eine Geschichte. Leitfaden zur Aktion Suchtvorbeugung in NRW, Köln (in immer neuen Auflagen) [zu bestellen über GINKO in Mühlheim/ Ruhr].

${ }^{31}$ Schneider W. Schnüffelstoffe: Schneller Rausch für wenig Geld. Suchtreport 1998; 5: 6-11

32 Larsen R. Anästhesie. München: Urban \& Fischer 2002

${ }^{33}$ Musharbash Y. Rausch aus dem Supermarkt. taz vom 27.8 .02 (s.: www.taz.de).

${ }^{34}$ drug scouts. Informationen zu Schnüffelstoffen: www.suchtzentrum.de/drugscouts/dsv3/stoff/schnueffelstoffe/html auch: LachgasSeite (Homepage im Internet ohne klar erkenntlichen Autor): www.kapsler.de

${ }^{35}$ S.B. [vermutlich $=$ Stephanie Bonin]. Minuten-Flug. JUBAZ 2002; 2: 8-10; dort auch diverse Internet-Adressen zur „Harmlosigkeit“ von Lachgas

${ }^{36}$ Polizei. www.netcologne.de/Vorbeugung.

37 Informationen zu „anderen“ Drogen, im Internet www.hammer hart.de, www.suchtzentrum.de/drugscout/dsv3/start.html, - als Buch z.B.: Lewin L. Phantastica. Die betäubenden und erregenden Genußmittel, Linden: Volksverlag, 1980 (= Reprint der 2. Auflage 1927), Parnefjord R. Das Drogentaschenbuch. Stuttgart: Thieme, 1997, Rätsch R. Enzyklopädie der psychoaktiven Substanzen. Stuttgart: Wiss. Verl. Ges./Aarau: AT Verlag 1998

${ }^{38}$ Schneider W. Lösungsmittelmißbrauch bei Heranwachsenden als Einstieg in die Drogensucht. Sozialpädiatrie 1990; 12: 900-905 